\title{
Interactive comment on "Large scale climate signals of a European oxygen isotope network from tree-rings - predominantly caused by ENSO teleconnections?" by Daniel F. Balting et al.
}

Daniel F. Balting et al.

daniel.balting@awi.de

Received and published: 22 September 2020

Our reply to reviewer 1 is attached as a supplement.

Please also note the supplement to this comment:

https://cp.copernicus.org/preprints/cp-2020-39/cp-2020-39-AC1-supplement.pdf 